\title{
Methods used to measure postoperative insulin resistance
}

\author{
Ozlem Korkmaz Dilmen ${ }^{1}$
}

Received: 11 April 2016 / Accepted: 12 May 2016 / Published online: 1 July 2016

(C) Springer Science+Business Media Dordrecht 2016

\section{Editor,}

I read with interest the recent article by Canbay et al. [1].

The authors investigated the effect of the preoperative oral carbohydrate (POC) treatment on development of the postoperative insulin resistance following radical prostatectomy, and they concluded that POC treatment reduces insulin resistance in the postoperative period. But they did not mention which method was used to measure insulin resistance. Various techniques are being used to analyze insulin resistance. Although the hyperinsulinemic normoglycemic clamp technique is the gold standard, its implementation is very difficult. The homeostatic model assessmentinsulin resistance equation [HOMA-IR $=$ fasting insulin $\left(\mu \mathrm{g} \mathrm{mL}^{-1}\right) \times$ fasting blood glucose $\left.\left(\mathrm{mg} \mathrm{dL}^{-1}\right) / 405\right]$, artificial pancreas with a closed loop system and quantitative insulin sensitivity check index are other methods that use to measure insulin resistance [2]. Besides this, due to insulin resistance not only occurring following starvation, prolonged fasting time, surgery and trauma, but also as a normal component of the diurnal rhythm, overnight fasting reduces insulin sensitivity before breakfast. Therefore, obtained blood samples timing should be clarified (morning or evening, etc.).

\section{References}

1. Canbay O, Adar S, Karagoz AH et al (2014) Effect of preoperative consumption of high carbohydrate drink (Pre-Op) on postoperative metabolic stress reaction in patients undergoing radical prostatectomy. Int Urol Nephrol 46:1329-1333

2. Bilku DK, Dennison AR, Hall TC et al (2014) Role of preoperative carbohydrate loading: a systematic review. Ann R Coll Surg Engl 96:15-22

This comment refers to the article available at doi:10.1007/s11255-013-0612-y.

Ozlem Korkmaz Dilmen

korkmazdilmen@gmail.com

1 Department of Anaesthesia and Intensive Care, Cerrahpasa

Medical Faculty, University of Istanbul, Cerrahpasa,

34098 Istanbul, Turkey 